\title{
SJRM \\ Comparison of the effectiveness of radical surgery with combination therapy (Sarem's Women Hospital protocol) in the management of severe endometriosis, a randomized controlled trial
}

\section{ARTICLE INFO}

\section{Article Type}

Clinical Tria

\section{Authors}

Abootaleb Saremi ${ }^{1}$, iD MD*

Afshin Fazel ${ }^{2}, \mathrm{MD}$

Mahboobeh Rasekhi ${ }^{3}$, MD

Mohammad Reza Nateghi

\section{${ }^{1}$ Sarem Fertility \& Infertility Research Center (SAFIR) \& Sarem Cell Research Center (SCRC), Sarem Women's Hospital, Iran University of Medical Sciences (IUMS), Tehran, Iran \\ 2 Lariboisière Hospital, Paris, France \\ ${ }^{3}$ Sarem Fertility \& Infertility Research Center (SAFIR) Sarem Women's Hospital, Iran University of Medical Sciences (IUMS), Tehran, Iran}

*Corresponding Author Address: Sarem Women Hospital, Basij Square, Phase 3, EkbatanTown, Tehran, Iran Postal code: 1396956111 Phone: +98 (21) 44670888 Fax: +98 (21) 44670432

Dr.saremi@sarem.org

\section{Article History}

Received: September 11, 2019 Accepted: November 05, 2019 e Published: December 17, 2020

\section{ABSTRACT}

Aim: This study was performed to compare the effectiveness of radical surgery and combination therapy (Sarem's Women Hospital protocol) and their costs in patients with grade IV (Severe) endometriosis.

Material and methods: In this randomized clinical trial, 44 patients with grade IV endometriosis were randomly divided into two groups. In the first group $(n=19)$, all endometriosis lesions were completely removed by radical surgery. In the second group ( $\mathrm{n}=25)$ no therapeutic surgery (except diagnosis) was performed and patients were included in the combination therapy treatment protocol. In this protocol (combination therapy), patients received 12 months of drug treatment with GnRH agonist (Decapeptyl $3.75 \mathrm{mg}$, IM Inj.) every 28 days. The fine adhesions or very poor remnants of endometriosis lesions removed by second-look laparoscopy. The severity of pelvic pain and dysmenorrhea was analyzed according to the VAS scale, one month and two years later, along with treatment costs in both groups.

Results: One month after the end of treatment, pelvic pain and dysmenorrhea were significantly lower in the combination therapy group, and on the other hand, two years after treatment, recurrence of dysmenorrhea and pelvic pain were significantly higher in the radical surgery group than in the combination therapy group. There were no post-treatment complications in the combination therapy group, while some patients in the radical surgery treatment group developed complications. Also in this study, it was shown that the cost of treatment in the radical surgery group was significantly higher than the combination therapy group.

Discussion and Conclusion: Combination treatment of endometriosis (Sarem's Women Hospital protocol) is more effective in reducing dysmenorrhea and pelvic pain one month and two years after treatment and with much fewer complications and is superior to radical surgery in terms of treatment costs.

Keywords: Endometriosis, Radical Surgery, Combination Therapy, Decapeptyl, GnRH Agonist, Dysmenorrhea, Pelvic Pain, Recurrence, Cost, Randomized Controlled Trial (RCT)

Copyright(C) 2020, ASP Ins. This open-access article is published under the terms of the Creative Commons Attribution-Noncommercial 4.0 International License which permits Share (copy and distribute the material in any medium or format) and Adapt (remix, transform, and build 
در كروه جراحى راديكال نسبت به كروه درمان تركيبى بيشتر بود. در كروه

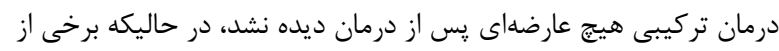

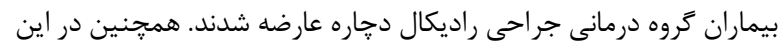

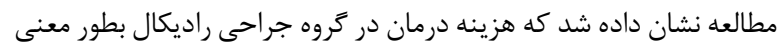

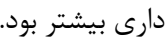
بحث و نتيجه

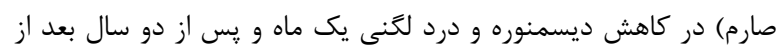

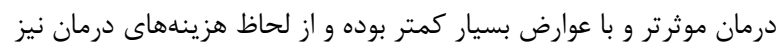
نسبت به جراحى راديكال برترى دارد.

كليد وازمها: اندومتريوز، جراحى راديكال، درمان تركيبى، دكايتيتل،

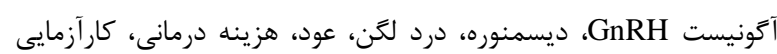

بالينى تصادفى

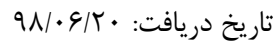

تاريخ يذيرش:

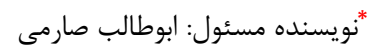

\section{مقدمه}

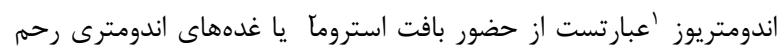

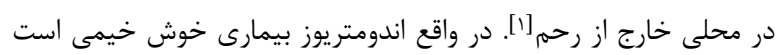

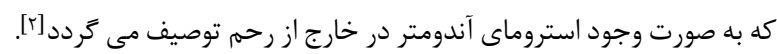

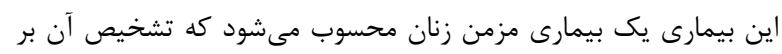

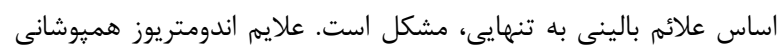

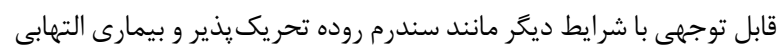

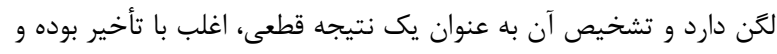

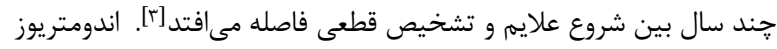

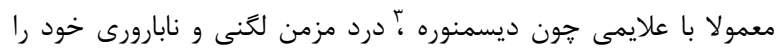

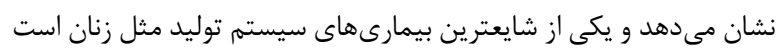

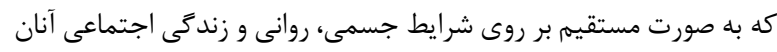

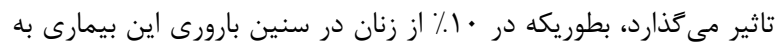

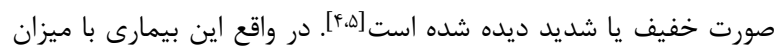

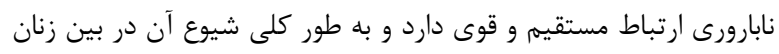

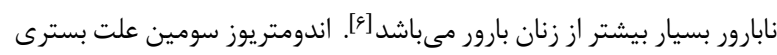

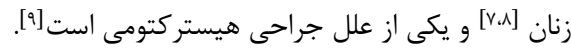

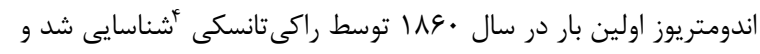

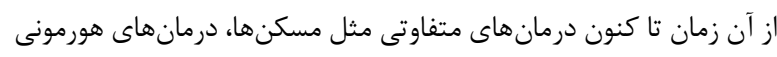

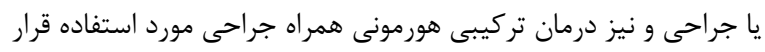

Dysmenorrhea $^{r}$ Karl Freiherr von Rokitansky
مقايسه اثر بخشى جراحى راديكال با روش درمان تركيبى (يروتكل بيمارستان صارم) در

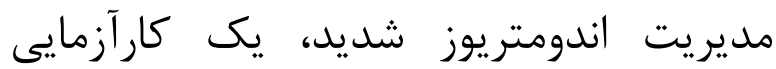

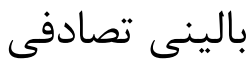

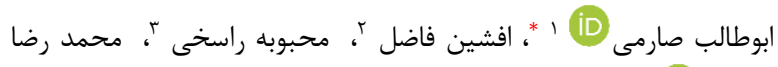
ناطقى أوطالب صارمى

' مركز تحقيقات بارورى و نابارورى صارم، مركز تحقيقات سلولى و مولكولى

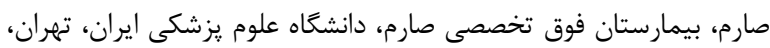
ايران r بيمارستان لارى بيوزر، خاريس، فرانسه

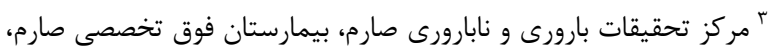

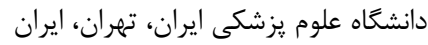

جكيده

اهداف: اين مطالعه به منظور مقايسه اثر بخشى جراحى راديكال و درمان

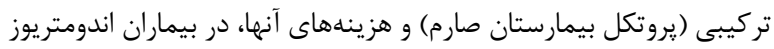

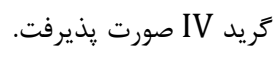

مواد و روش ها: در اين كارآزمايى بالينى تصادفى، بf بf بيمار با تشخيص

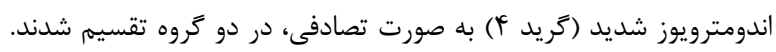

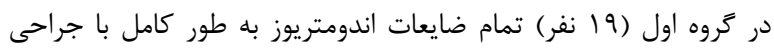

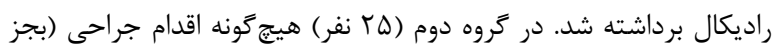

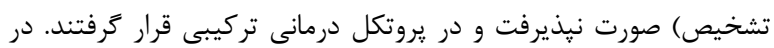

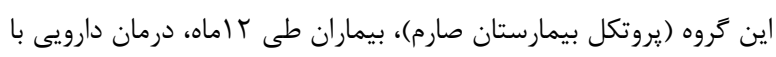

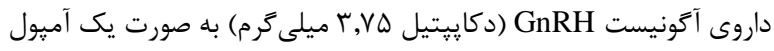

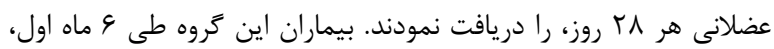

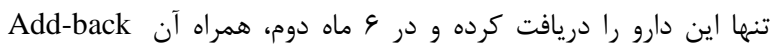

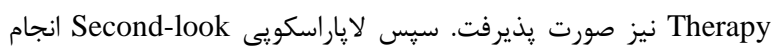

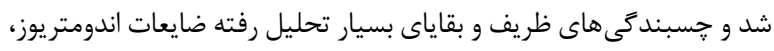

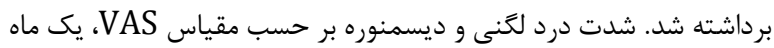

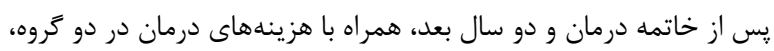
تجزيه و تحليل گرديد.

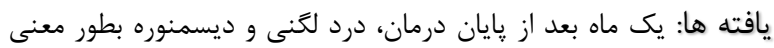

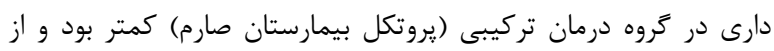

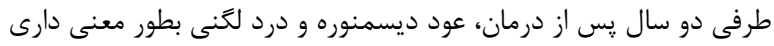

Endometriosis' Endometrial Stromal Tissue ${ }^{r}$

دانشنامه صارم در طب بارورى

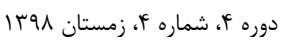




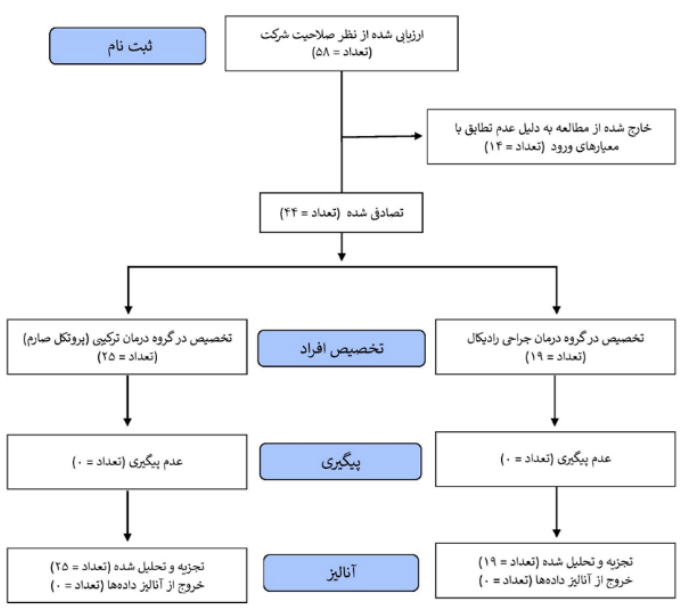

نمودار شماره ا. جريان روند مطالعه (كنسرت +1.1)

در تروه درمانى جراحى راديكال، 19 بيمار، تحت جراحى لإياراسكوبى (ريى

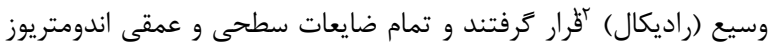

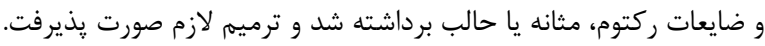

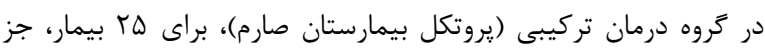

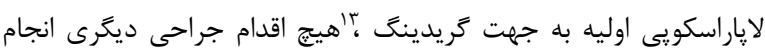

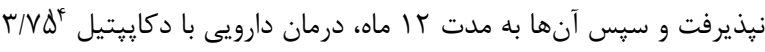

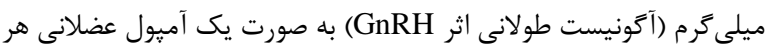

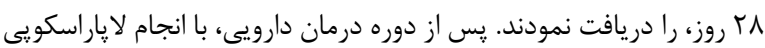

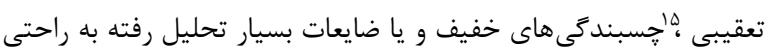

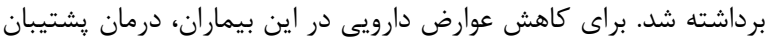

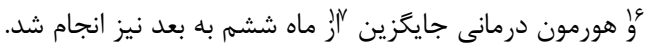

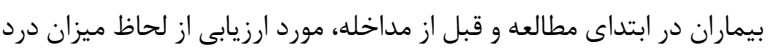

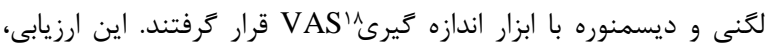

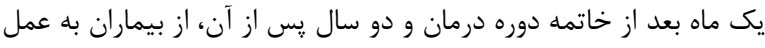

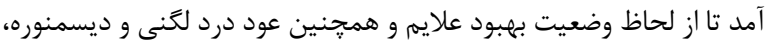
ارزيابى و مقايسه بين دو گروه انجام شود.

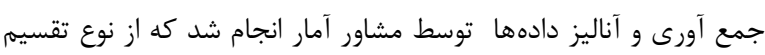

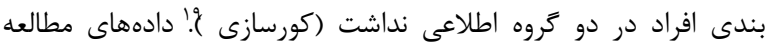

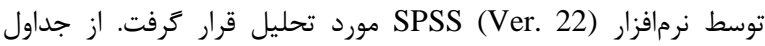

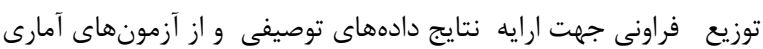

Radical Surgery'r Grading 't' Decapeptyl (Triptorelin Pamoate)' Second-Look Laparoscopy' Add-Back Therapy' Hormone Replacement Therapy (HRT) '" Visual Analogue Scale (VAS) ${ }^{1 \wedge}$ Blindness'
كرفتهاند، ولى تاكنون هيج درمان قطعى براى آن يافت نشده است[1(1.]]

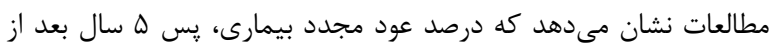

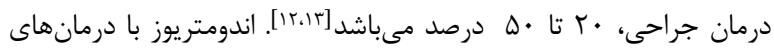

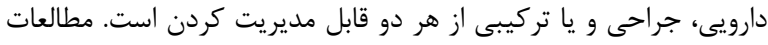

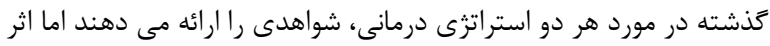

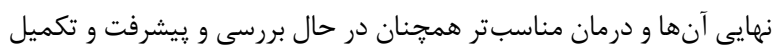

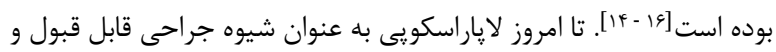

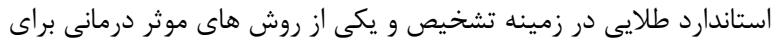

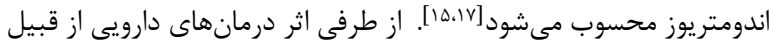

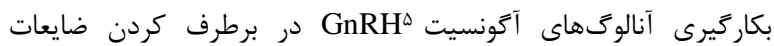

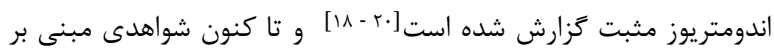

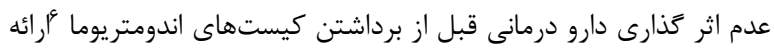

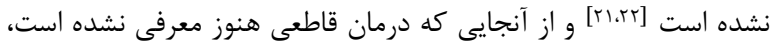

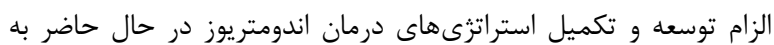
خوبى احساس مىشود.

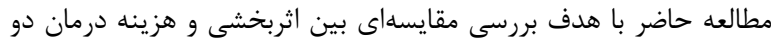

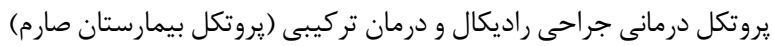

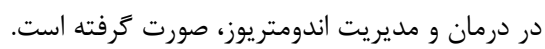

\section{مواد و روشها - ماد}

در اين مطالعه كارآزمايى بالينى تصادفى ؛ تعداد بf ب بيمار با اندومتريوز

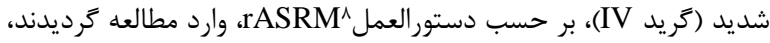

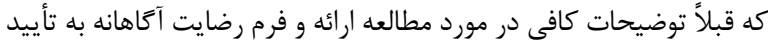

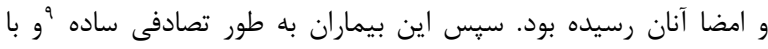

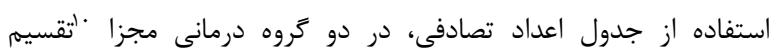

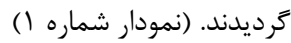

Gonadotropin Release Hormone (GnRH) Agonist (GnRHa)

Endometrioma Randomized Clinical Trial (RCT) The revised American Society for Reproductive Medicine (rASRM)

Simple Random Sampling Parallel Group Study' CONSORT Flow Diagram of RCT ${ }^{\prime \prime}$

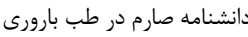

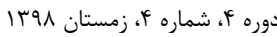


جدول شماره Y. مقايسه وضعيت تأهل، باردارى، تولد نوزاد زنده و سقط

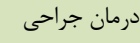

راديكال

(19)

تعداد (درصد)

$($ (L.T.\%.) $\triangle$

$(V r . V /) \mid. \mathcal{F}$

$(\lambda \%) r$

وضعيت تأهل * مجرد

$(9 T \%) r r$

مجرد

متأهل

\begin{tabular}{|c|c|}
\hline$(\Delta V .9 \%) 11$ & $(\Delta \varphi . \%) \backslash F$ \\
\hline$(\ulcorner\varsigma . \Lambda \%) \vee$ & $(\kappa \cdot . \%) 1$. \\
\hline$(\Delta . r \%) ।$ & $(\kappa . \%) 1$ \\
\hline
\end{tabular}

* باردارى

$(r \& . A \%) \mathrm{V}$

$(\boldsymbol{F} \cdot \%) \mid$

صفر

دوم

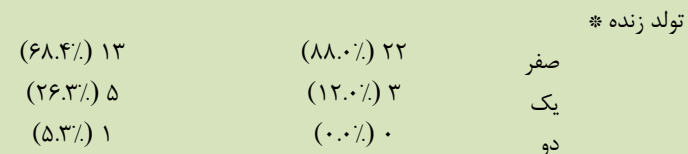

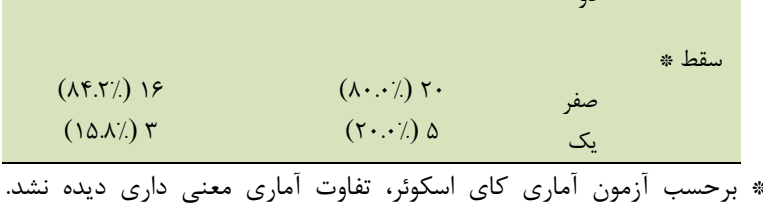

(PV>0.05)

برحسب دادههاى بدست آمده از مقياس اندازهخيرى VAS (در بازه صفر تا

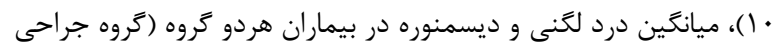

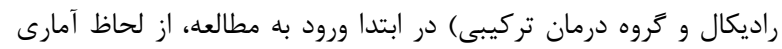

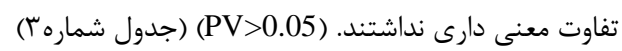

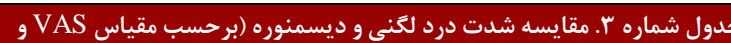

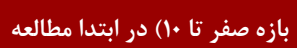

\begin{tabular}{|c|c|c|c|}
\hline ميانكَينها & $\begin{array}{c}\text { درمان جراحيكال } \\
\text { راديكال } \\
\text { (9 نفر) }\end{array}$ & 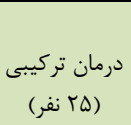 & يارامتر \\
\hline (مقادير & ميانكَين & ميانكين & \\
\hline \multirow[t]{2}{*}{ منفى) } & (انحر اف معيار) & (انحراف & \\
\hline & & معيار) & \\
\hline . .99 & $(\cdot . f \Delta) \wedge . \Delta \cdot$ & $(\cdot . \digamma r) \wedge . r$. & درد لحنى \\
\hline$(\cdot . r F)$ & r. & (.) & ديسمنور \\
\hline
\end{tabular}

Fisher's Exact Test ${ }^{r r}$

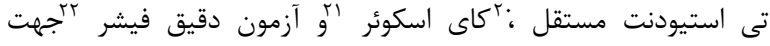

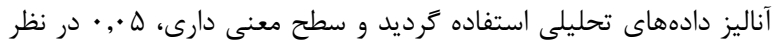

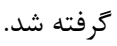

\section{نتايج}

در اين مطالعه 19 بيمار زن مبتلا به اندومتريوز متوسط تا شديد با ميانخين

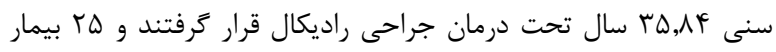

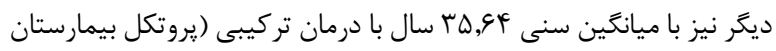

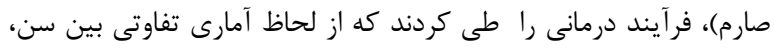
قد، وزن و شاخص توده بدنى دو كروه ديده نشد (PV>0.05) (PV). (جدول

شماره ()

\begin{tabular}{|c|c|c|c|}
\hline \multicolumn{4}{|c|}{ جدول شماره ا. مقايسه سن، قد، وزن و شاخص توده بدنى } \\
\hline تفاضل ميانكينها & $\begin{array}{c}\text { درمان جراحى } \\
\text { راديكال } \\
\text { (19 نفر) }\end{array}$ & 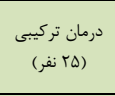 & بارارتر \\
\hline (مقادير منفى) & $\begin{array}{c}\text { ميانكين (انحراف } \\
\text { معيار) }\end{array}$ & $\begin{array}{l}\text { ميانكين (انحراف } \\
\text { معيار) }\end{array}$ & \\
\hline . r. & $(r \cdot \Lambda \cdot) r \Delta . \wedge f$ & $(\Delta . r) r \Delta . \varphi^{f}$ & سن (سال)* \\
\hline$\cdot \Delta \Lambda$ & $(\Delta . \Delta r)|8| . \Delta \wedge$ & 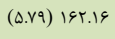 & قد (سانتىمتر) : \\
\hline r. .9 & $(11.1 f) \varepsilon \Delta . V F$ & (11.v• $9 r .91$ & 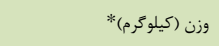 \\
\hline. .94 & $(f . \lambda f) r \Delta .1 f$ & $(F . \cdot \varphi)$ rF.r. & شربع) "* توده بدنى (كيلوكرم/متر \\
\hline
\end{tabular}

* برحسب آزمون آمارى تى استيودنت مستقل، تفاوت آمارى معنى دارى ديده نشد. ( $\mathrm{PV}>0.05)$

^ درصد زنان تروه درمانى تركيبى، مجرد و rو در دصد آنها متاهل بودند.

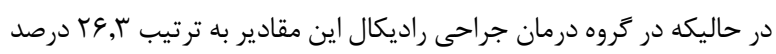

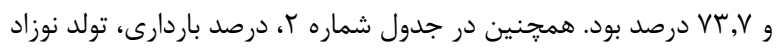

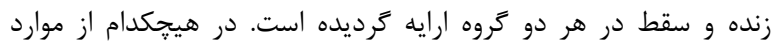

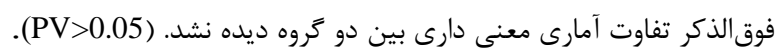

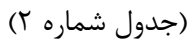

Independent Samples T-Test ${ }^{r}$. Chi Square Test ${ }^{r /}$

دانشنامه صارم در طب بارورى 


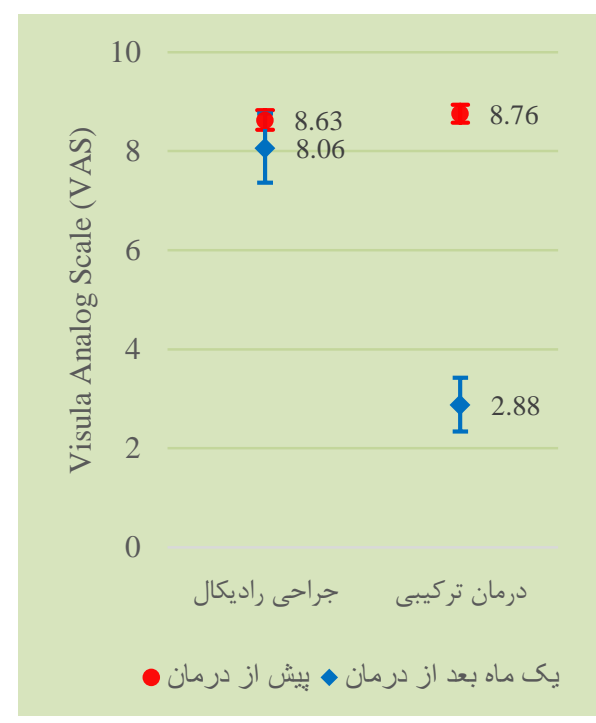

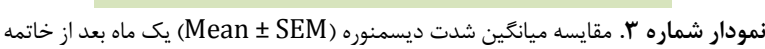
درمان (PV)

جهت بررسى ميزان عود علايم اندومتريوز و مقايسه آن ميان دو گروه

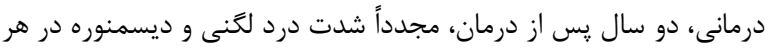

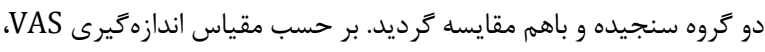

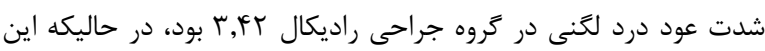

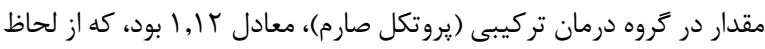
آمارى تفاوت معنى دارى بين دو كروه ديده شد در (

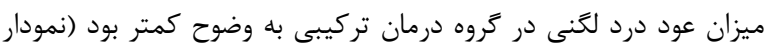

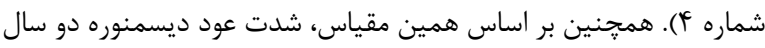

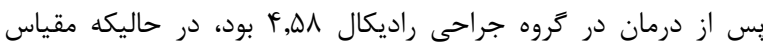

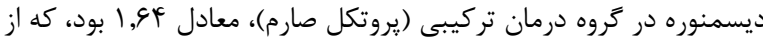

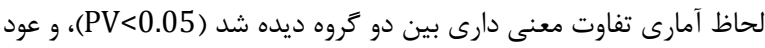

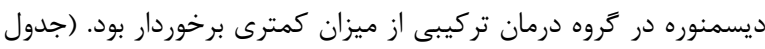

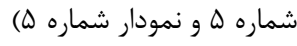

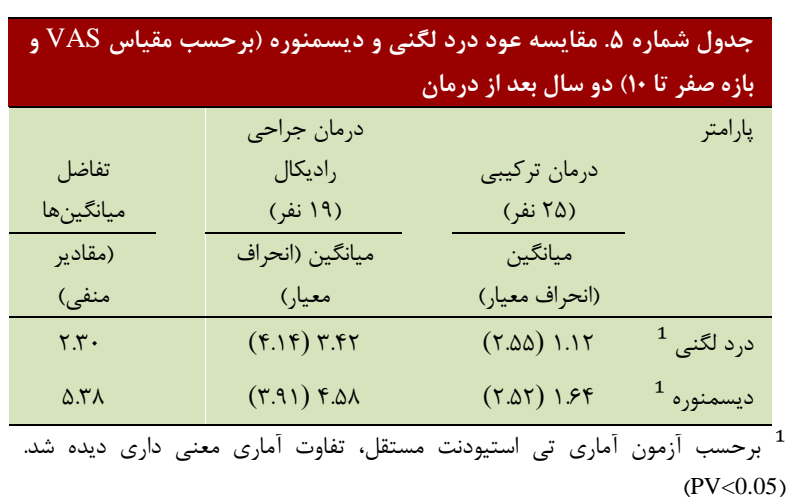

برحسب دادههاى بدست آمده از مقياس اندازهخيرى VAS (در بازه صفر

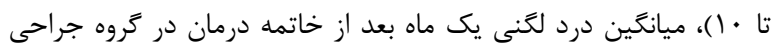

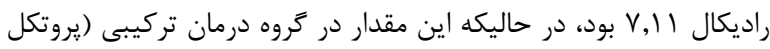

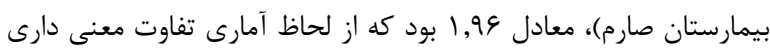

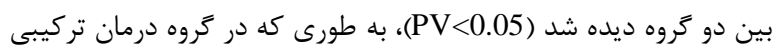

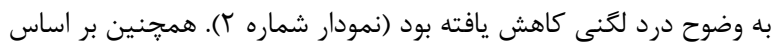

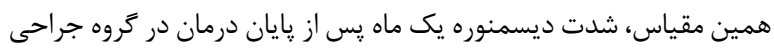

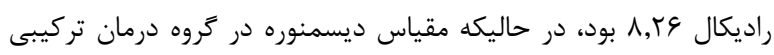

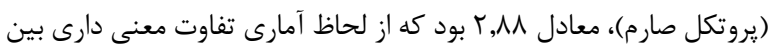

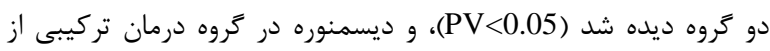

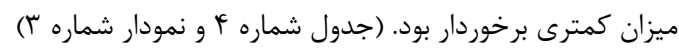

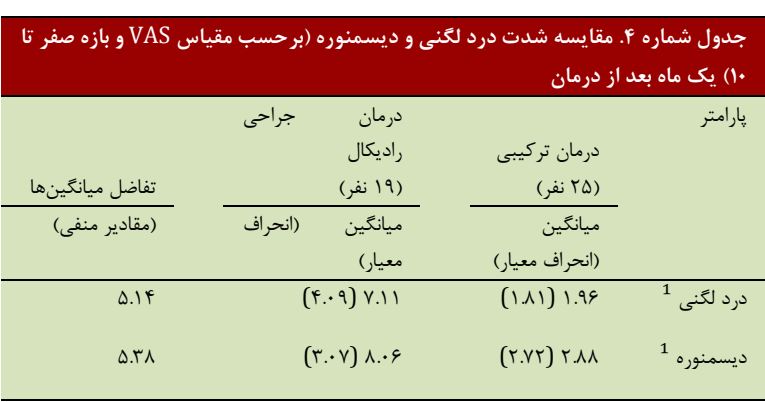

1 برحسب آزمون آمارى تى استيودنت مستقل، تفاوت آمارى معنى دارى ديده شد. (PV) (PV)

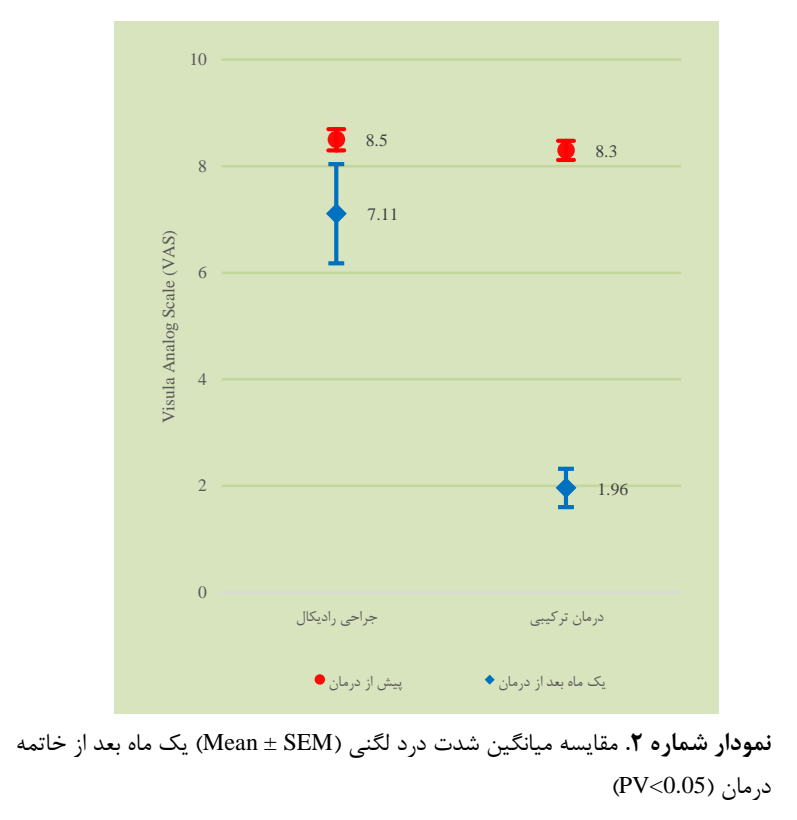

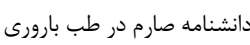




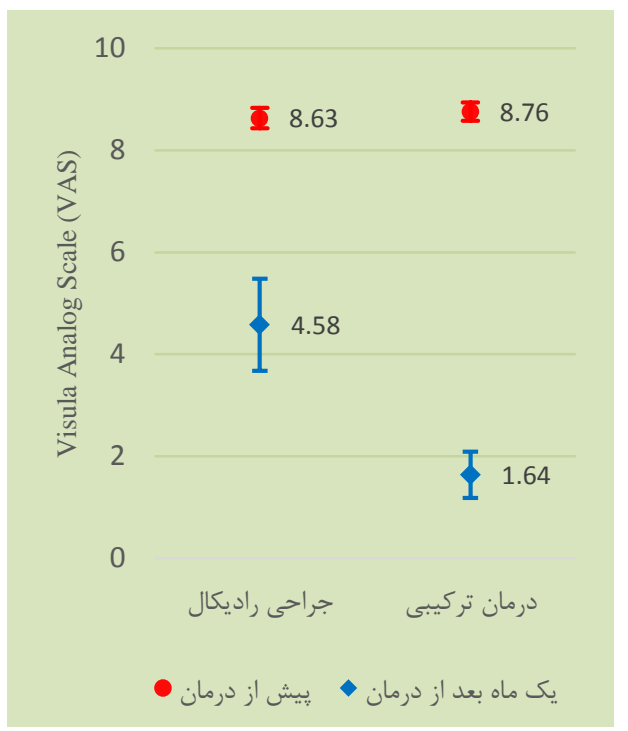

نمودار شماره ه. مقايسه ميانگين شدت ديسمنوره (Mean I SEM) دو سال بعد از درمان $(\mathrm{PV}<0.05)$

در نهايت از لحاظ هزينههاى درمان، گروه درمان تركيبى (يروتكل

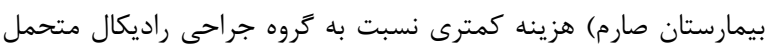

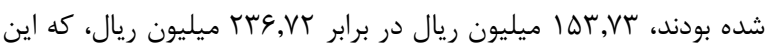

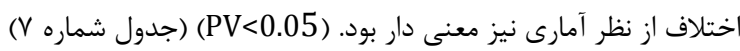

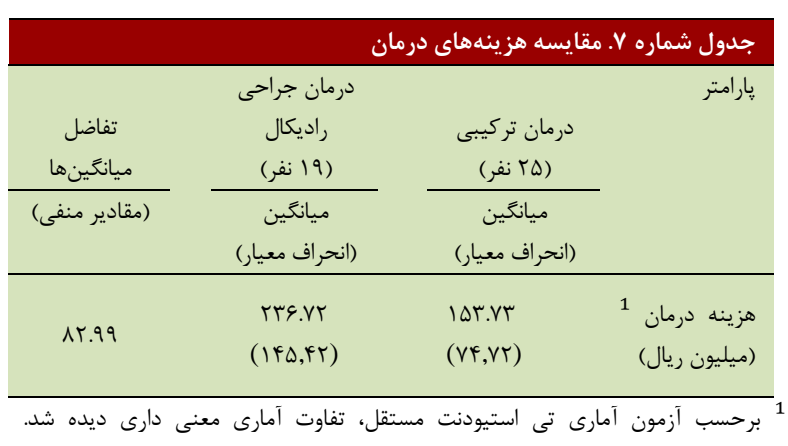

$(\mathrm{PV}<0.05)$

بحث:

نتايج اين مطالعه كارآزمايى بالينى تصادفى شده، نشان داد كه درمان

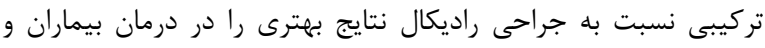

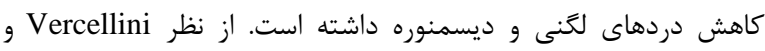

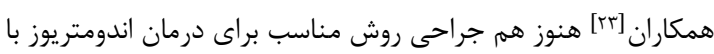

Colostomy ${ }^{\text {ro }}$ Vaginal Fistula ${ }^{r-}$

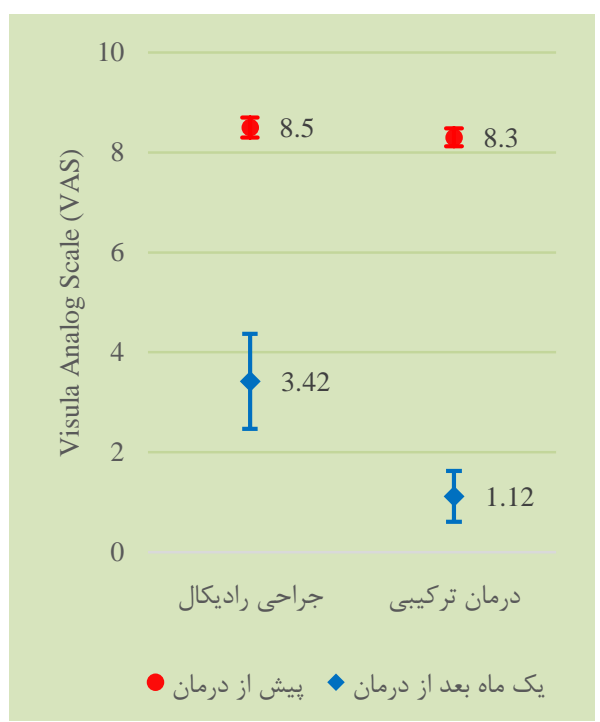

نمودار شماره F. مقايسه ميانكَين شدت درد لكَنى (Mean I SEM) دو سال بعد از درمان $(\mathrm{PV}<0.05)$

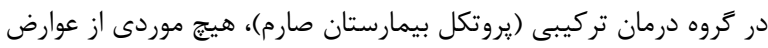

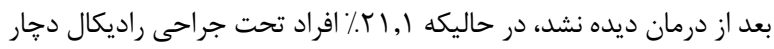

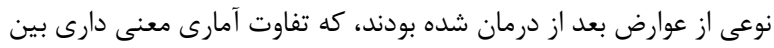

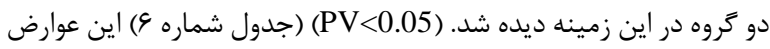

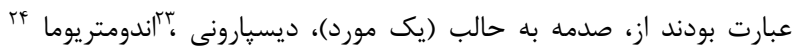

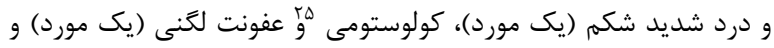
فيستول وارينال ؤهمراه بى اختيارى ادرار (يك مورد).

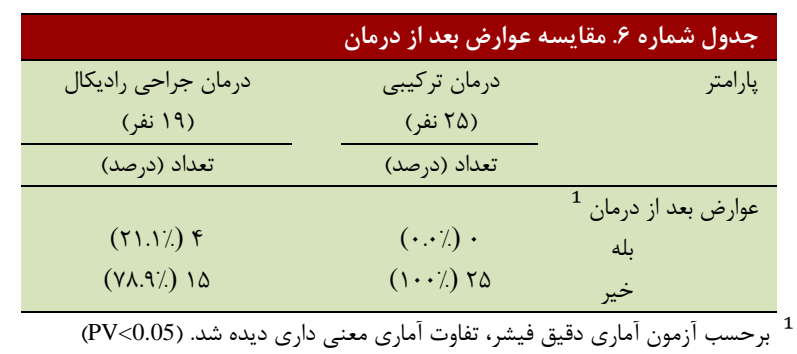

Dyspareunia $^{r+}$ Endometrioma ${ }^{\text {rs }}$

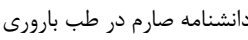




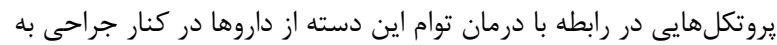

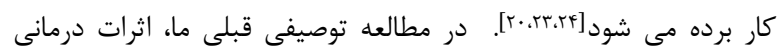

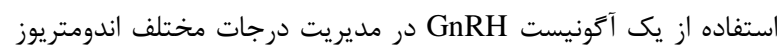

كزارش كرديده است [19. Schenken

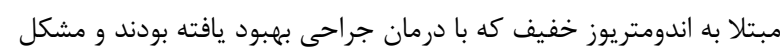

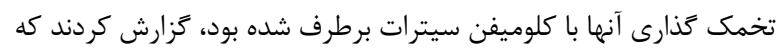

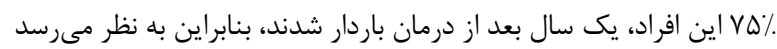

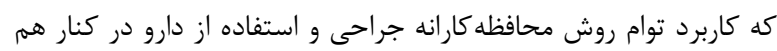

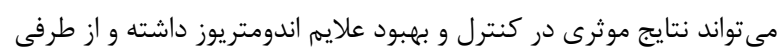
سبب حفظ قدرت بارورى نيز كرديده است.

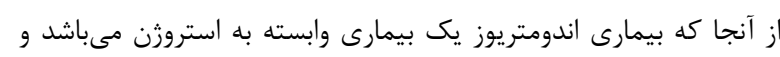

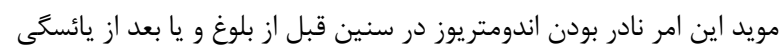

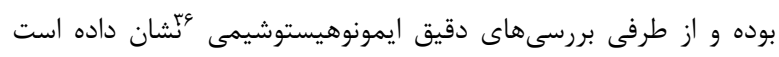

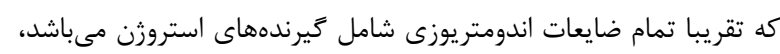

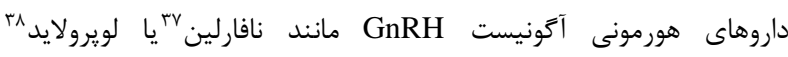

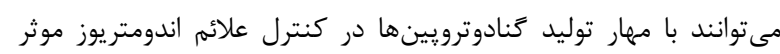

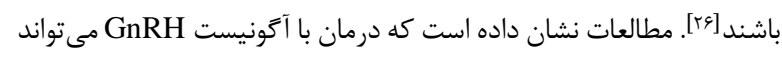

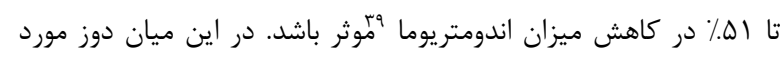

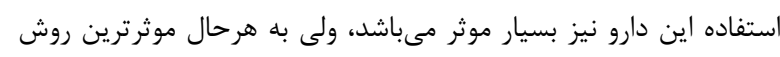

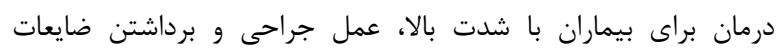

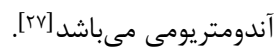

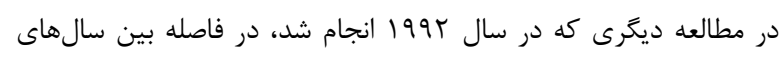

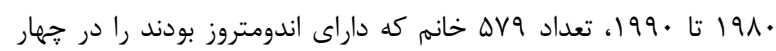

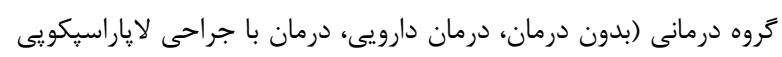

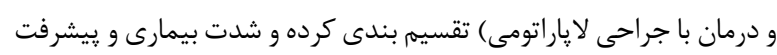

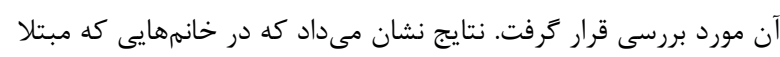

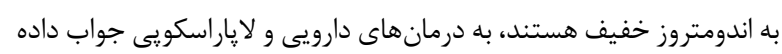

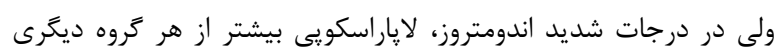

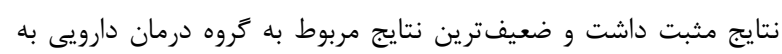

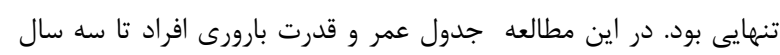

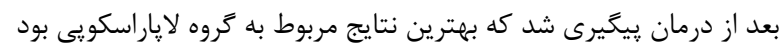

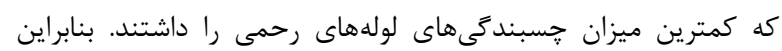

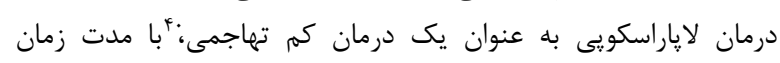

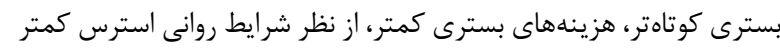

Add-Back Therapy ${ }^{r s}$ Hormone Replacement Therapy (HRT) ${ }^{\text {ro }}$ Immunohistochemistry (IHC) ${ }^{r r}$ Nafarelin (Synarel) $)^{r v}$ Leuprolide (Leuprorelin, Lupron) ${ }^{r \wedge}$ Endometrioma $^{\text {rq }}$ Minimally Invasive Procedure ${ }^{\varepsilon}$
كمترين شانس عود مجدد مىباشد، اما از طرفى نيز ديده شده است كه

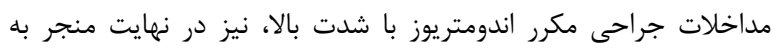

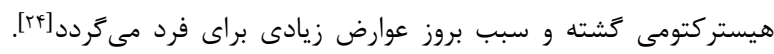

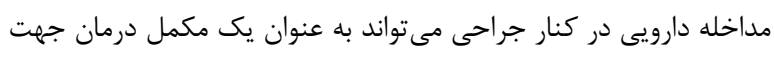

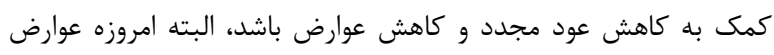

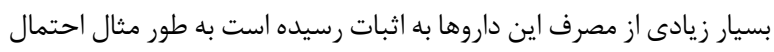

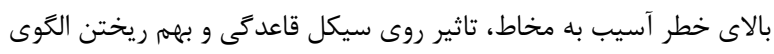

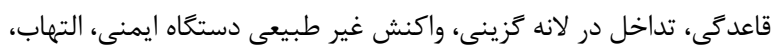

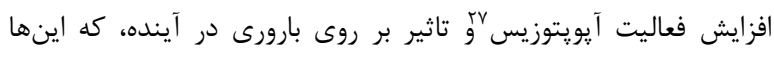

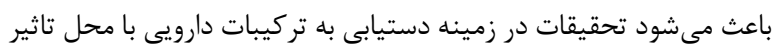

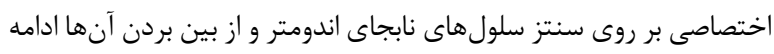

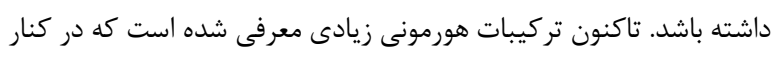

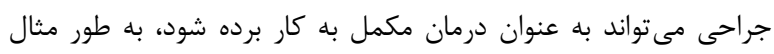

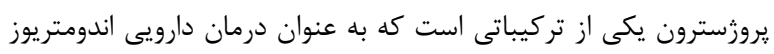

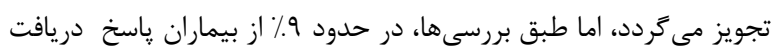

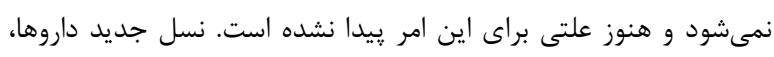

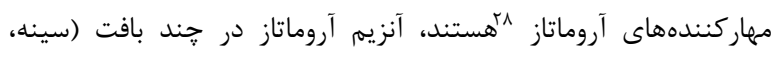

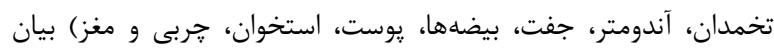

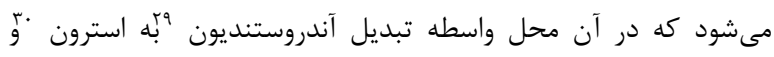

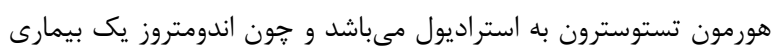

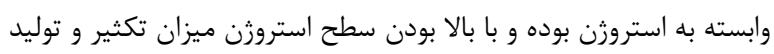

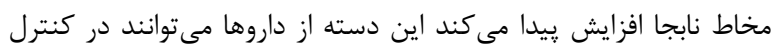

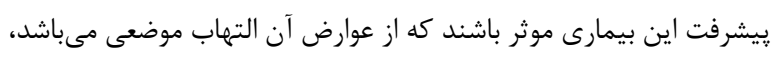

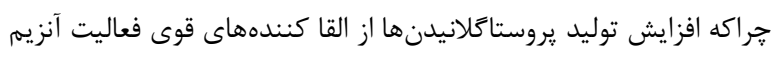

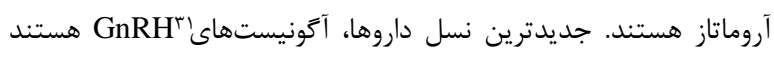

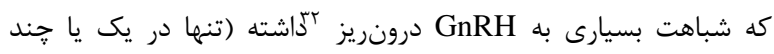
استخلاف جانبى آمينو اسيد تفاوت دارند) كه با كاهش فعاليت محور

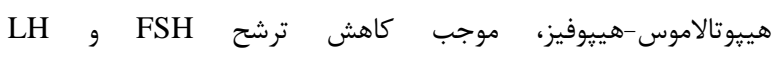
از هييوفيز مىشوند. عدم ترشح اين دو هورمون موجب مهار مهار ترشح

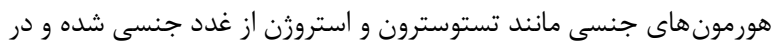

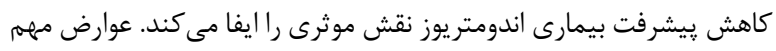

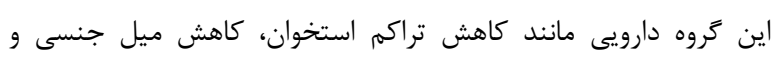

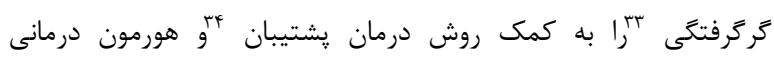

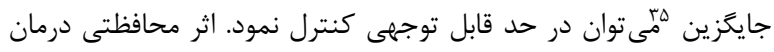

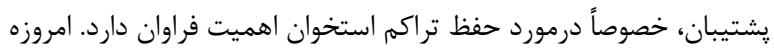




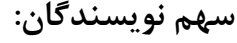

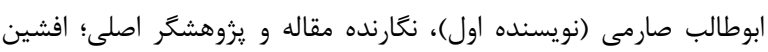

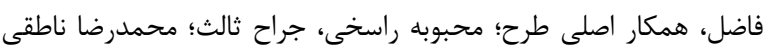

$$
\begin{aligned}
& \text { (روش شناس و تحليل }
\end{aligned}
$$

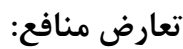

$$
\begin{aligned}
& \text { در اين مطالعه تعارض منافع وجود نداشت. }
\end{aligned}
$$

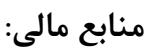

$$
\begin{aligned}
& \text { هزينه هاى يزوهش توسط مركز تحقيقات بارورى و نابارورى صارم تامين } \\
& \text { شده است. } \\
& \text { منابع }
\end{aligned}
$$

1. Tsoumpou I, Kyrgiou M, Gelbaya TA, Nardo LG. The effect of surgical treatment for endometrioma on in vitro fertilization outcomes: a systematic review and meta-analysis. Fertil Steril. 2009 Jul 1;92(1):75-87.

2. Fritz MA, Speroff L. Clinical Gynecologic Endocrinology and Infertility. Lippincott Williams \& Wilkins; 2012. 1488 p.

3. Kennedy S, Bergqvist A, Chapron C, D'Hooghe T, Dunselman G, Greb R, et al. ESHRE guideline for the diagnosis and treatment of endometriosis. Hum Reprod [Internet]. 2005 Oct 1 [cited 2020 Dec 1];20(10):2698-704. Available from: https://pubmed.ncbi.nlm.nih.gov/15980014/

4. Engel J, Berkes E, Tinneberg HR. Klassifikation der Endometriose. Gynakologe. 2015;48(3):200-8.

5. Simoens S, Hummelshoj L, D'Hooghe T. Endometriosis: cost estimates and methodological perspective. Hum Reprod Update [Internet]. $2007 \mathrm{Jul}$ 1;13(4):395-404. Available from: https://academic.oup.com/humupd/article/13/4/3 95/2457867

6. Lin XN, Wei ML, Tong XM, Xu WH, Zhou F, Huang QX, et al. Outcome of in vitro fertilization in endometriosis-associated infertility: A 5-year database cohort study. Chin Med J (Engl). 2012;125(15):2688-93.

7. Velebil P, Wingo PA, Xia Z, Wilcox LS, Peterson HB. Rate of hospitalization for gynecologic disorders among reproductive-age women in the United States. Obstet Gynecol [Internet]. 1995 [cited 2020 Dec 1];86(5):764-9. Available from: https://pubmed.ncbi.nlm.nih.gov/7566845/
و زمان بهبودى كوتاهتر نسبت به ساير روشها بِيشنهاد كرديد [^مَ].

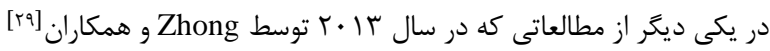

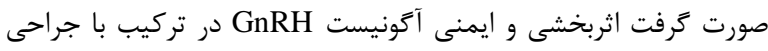

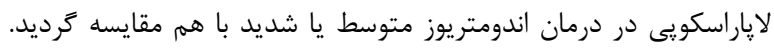

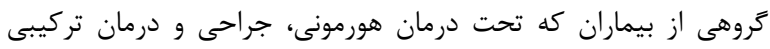

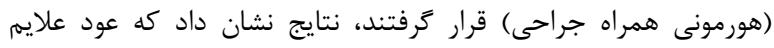

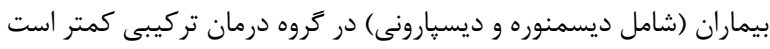

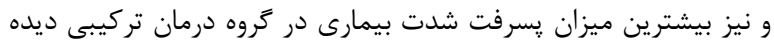

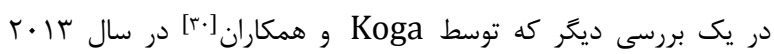

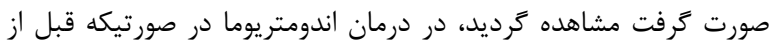

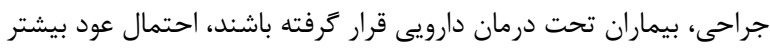

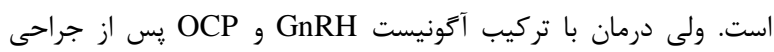

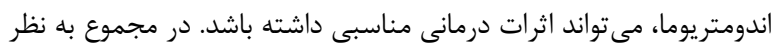

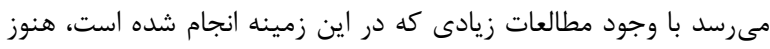
يروتكلى واحد و قابل تعميم معرفى نشده است.

\section{نتيجه كيرى}

در تحقيق حاضر اثربخشى، عوارض و هزينه دو روش مختلف درمان در

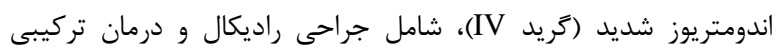

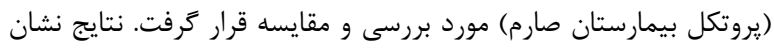

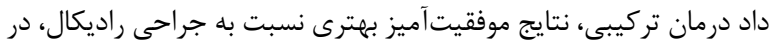

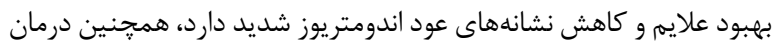

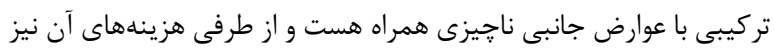

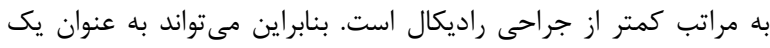

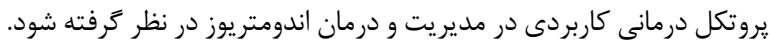

$$
\text { تشكر و قدردانى: }
$$

از لطف بىدريغ كاركنان محترم بيمارستان و مركز تحقيقات بارورى و و وانئ

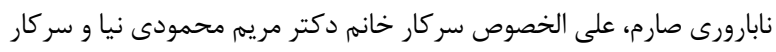

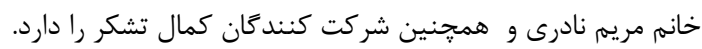

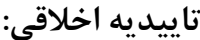

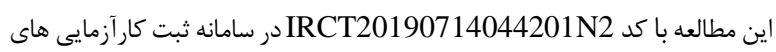

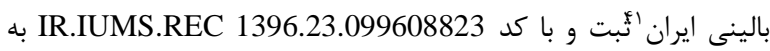
تاييديه كميته اخلاقى دانشكاه علوم يزشكى ايران رسيده است.

Iranian Registry for Clinical Trials (IRCT) $)^{\natural /}$ 
مقايسه اثر بخشى جراحى راديكال با روش درمان تركيبى (يروتكل بيمارستان صارم) در مديريت اندومتريوز شديد با ب

https://linkinghub.elsevier.com/retrieve/pii/S0015 028203001559

18. Vercellini P, Trespidi L, Colombo A, Vendola N, Marchini M, Giorgio Crosignani P. A gonadotropinreleasing hormone agonist versus a low-dose oral contraceptive for pelvic pain associated with endometriosis*t. Vol. 60, Fertility and Sterility. 1993. 19. Brown J, Pan A, Hart RJ. Gonadotrophin-releasing hormone analogues for pain associated with endometriosis. Cochrane database Syst Rev [Internet]. 2010 Dec 8 [cited 2020 Dec 2];(12):CD008475. Available from: https://www.cochranelibrary.com/cdsr/doi/10.100 2/14651858.CD008475.pub2/full

20. Saremi AT, Pooladi A. Endometriosis management; A survey on medical \& laparoscopic treatment. Sarem J Reprod Med [Internet]. 2017 Oct 1;2(4):147-51. Available from: http://saremjrm.com/article-1-68-en.html

21. Hornstein MD, Yuzpe AA, Burry KA, Heinrichs LR, Buttram VL, Orwoll ES. Prospective randomized double-blind trial of 3 versus 6 months of nafarelin therapy for endometriosis associated pelvic pain. Fertil Steril [Internet]. 1995 Jan 1 [cited 2020 Dec 2];63(5):955-62. Available from: https://ohsu.pure.elsevier.com/en/publications/pro spective-randomized-double-blind-trial-of-3-versus6-months-of-2

22. Furness S, Yap C, Farquhar C, Cheong YC. Pre and post-operative medical therapy for endometriosis surgery. Cochrane Database Syst Rev [Internet]. 2004 Jul 19 [cited 2020 Dec 2];2004(3). Available from: /pmc/articles/PMC6984629/?report=abstract

23. Vercellini $P$, Crosignani $P$, Somigliana E, Vigan $P$, Frattaruolo MP, Fedele L. "Waiting for Godot": A commonsense approach to the medical treatment of endometriosis. Hum Reprod. 2011;26(1):3-13.

24. Bedaiwy MA, Barker NM. Evidence based surgical management of endometriosis. Middle East Fertil Soc J. 2012 Mar;17(1):57-60.

25. Schenken RS, Malinak LR. Conservative surgery versus expectant management for the infertile patient with mild endometriosis. Fertil Steril [Internet]. 1982 Feb [cited 2020 Dec 2];37(2):183-6. Available from: https://linkinghub.elsevier.com/retrieve/pii/S0015 02821646036X

26. Barbieri RL. Hormone treatment of endometriosis: The estrogen threshold hypothesis. Am J Obstet Gynecol. 1992 Feb 1;166(2):740-5.

27. Jacobson TZ, Duffy JMN, Barlow DH, Koninckx PR, Garry R. Laparoscopic surgery for pelvic pain associated with endometriosis. Vol. 2014, Cochrane
8. Whiteman MK, Kuklina E, Jamieson DJ, Hillis SD, Marchbanks PA. Inpatient hospitalization for gynecologic disorders in the United States. Am J Obstet Gynecol [Internet]. 2010 Jun [cited 2020 Dec 2];202(6):541.e1-541.e6. Available from: https://pubmed.ncbi.nlm.nih.gov/20132921/

9. Mounsey AL, Slawson DC. Diagnosis and Management of Endometriosis [Internet]. Vol. 74, American Family Physician. 2006 Aug [cited 2020 Dec 2]. Available from: www.aafp.org/afp.

10. Nezhat C, Nezhat F, Nezhat C. Endometriosis: ancient disease, ancient treatments. Fertil Steril [Internet]. 2012 Dec [cited 2020 Dec 2];98(6):S1-62. Available from: https://pubmed.ncbi.nlm.nih.gov/23084567/

11. Benagiano G, Brosens I, Lippi D. The History of Endometriosis. Gynecol Obstet Invest [Internet]. 2014 [cited 2020 Dec 2];78(1):1-9. Available from: https://www.karger.com/Article/FullText/358919 12. Vercellini P, Barbara G, Abbiati A, Somigliana E, Viganò $\mathrm{P}$, Fedele L. Repetitive surgery for recurrent symptomatic endometriosis: What to do? Vol. 146, European Journal of Obstetrics and Gynecology and Reproductive Biology. Elsevier Ireland Ltd; 2009. p. 15-21.

13. Rogers PAW, Adamson GD, Al-Jefout M, Becker CM, D'hooghe TM, Dunselman GAJ, et al. Research Priorities for Endometriosis: Recommendations From a Global Consortium of Investigators in Endometriosis. Reprod Sci. 2017;24(2):202-26.

14. Leyland N, Casper R, Laberge P, Singh S. Endometriosis: Diagnosis and management. J Obstet Gynaecol Can. 2010 Jul 1;32:S1-32.

15. Wykes CB, Clark TJ, Khan KS. REVIEW: Accuracy of laparoscopy in the diagnosis of endometriosis: a systematic quantitative review. BJOG An Int J Obstet Gynaecol [Internet]. 2004 Oct 19 [cited 2020 Dec 2];111(11):1204-12. Available from: https://pubmed.ncbi.nlm.nih.gov/15521864/

16. Saremi AT, Eshkevari AL, Pour KE, Pooladi A. Efficacy Analysis of an Experience on Development of the Combination Therapy Protocol for Endometriosis Management. Open J Obstet Gynecol [Internet]. 2017 [cited 2020 Dec 10];07(01):31-40. Available from: http://dx.doi.org/10.4236/ojog.2017.71004http://c reativecommons.org/licenses/by/4.0/

17. Stratton P. Diagnostic accuracy of laparoscopy, magnetic resonance imaging, and histopathologic examination for the detection of endometriosis. Fertil Steril [Internet]. 2003 May 1 [cited 2020 Dec 2];79(5):1078-85. Available from: 
Database of Systematic Reviews. John Wiley and Sons Ltd; 2014.

28. Adamson GD, Hurd SJ, Pasta DJ, Rodriguez BD. Laparoscopic endometriosis treatment: Is it better? In: Fertility and Sterility. Elsevier; 1993. p. 35-44.

29. Zhong Y, Zhang W, Zhang W, Cheng J, Lü Q, Zeng K. [Efficacy and safety of GnRH-a combine with laparoscope conservative surgery in the treatment of the moderate or severe endometriosis]. Zhonghua Fu Chan Ke Za Zhi [Internet]. 2013 Mar 1 [cited 2020 Dec 2];48(3):180-2. Available from: https://europepmc.org/article/med/23849939

30. Koga K, Osuga Y, Takemura Y, Takamura M, Taketani Y. Recurrence of endometrioma after laparoscopic excision and its prevention by medical management. Front Biosci - Elit [Internet]. 2013 Jan 1 [cited 2020 Dec 2];5 E(2):676-83. Available from: http://europepmc.org/article/med/23277022 